\title{
Lobectomy with sleeve resection in the treatment of bronchial tumours
}

\author{
G. M. REES and M. PANETH \\ Brompton Hospital, London, S.W.3
}

Forty-six patients with malignant tumours involving the orifices of the lobar bronchi were treated by lobectomy with sleeve resection of the bronchus. The results indicate that this procedure has many advantages when compared with pneumonectomy, which is the commonly practised alternative method of treatment. An immediate mortality rate following operation of $2 \cdot 2 \%$ and a five-year survival rate of $35 \%$ are recorded. Simple lung function tests taken before and after surgery in a small unselected group showed that operation caused little physiological impairment.

Bronchial carcinoma, situated at the level of the upper lobe orifices, may be treated surgically by pneumonectomy or lobectomy combined with sleeve resection of the main bronchus and end-toend anastomosis of the proximal and distal stumps, thus conserving the remainder of the lung. Many authors have emphasized the safety and wider applicability of sleeve resection. This procedure has a particular place in the management of patients with impaired respiratory function due to pulmonary or cardiac disease and also in older patients in whom pneumonectomy carries an unduly high operative risk (Price Thomas, 1956 ; Johnston and Jones, 1959 ; Le Roux, 1968).

We report 46 patients with malignant disease involving the upper or lower lobe orifices treated by sleeve resection by one surgeon (M. P.) at the Brompton Hospital during a 10-year period (19591969). They have been followed up at frequent intervals with repeated clinical and radiological assessments. Simple respiratory function tests have been carried out pre- and post-operatively in a small group of randomly selected patients to investigate the extent to which they are impaired by surgery.

\section{MATERIALS AND METHODS}

Forty patients were men and six were women. Their age distribution (Fig. 1) is typical of the distribution of bronchial carcinoma in the general population with a maximal incidence in the two decades 50-69. Some were over 70 years of age and many showed evidence of associated lung disease such as chronic bronchitis and emphysema.

Bronchoscopic evidence of tumour involvement of the upper lobe orifice was taken as an indication that

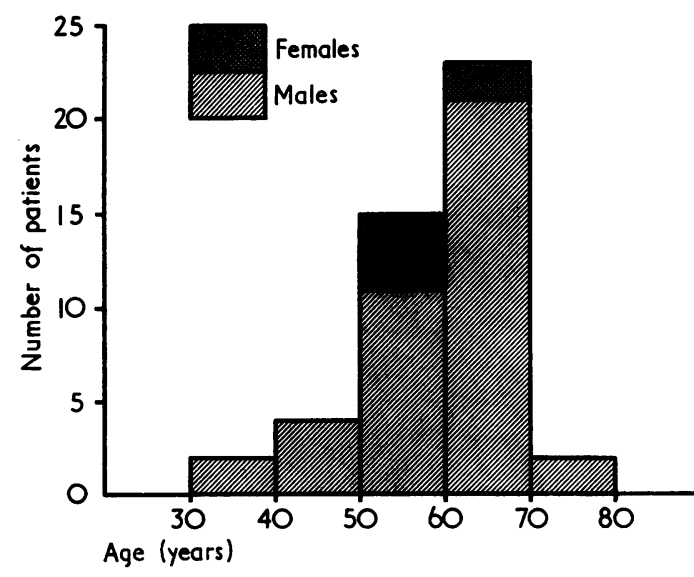

FIG. 1. Distribution of patients by age and sex.

the operative procedure should be sleeve resection, if technically possible, rather than pneumonectomy $\frac{D}{2}$ The surgical technique employed is in many wayssimilar to the one we use for lobectomy combined N with block dissection of the superior mediastinum to remove the lymphatic drainage of the upper lobeso (Brock and Whytehead, 1955; Nohl, 1962). AN postero-lateral incision is used, opening the chest ato the lower border of the fifth rib. A preliminary survey is then made to assess operability. The superior pulmonary vein is ligated as a first step, but on thes right side care is taken to preserve the venous drain age of the middle lobe. The fissure is then developed and branches of the pulmonary artery to the upper lobe are identified and ligated. Block dissection of? the superior mediastinum is carried out, severing the vagus nerve below the recurrent branch but leavinge the phrenic intact (Figs 2 and 3). On the right side the azygos vein is ligated and divided. The main bronchuso 


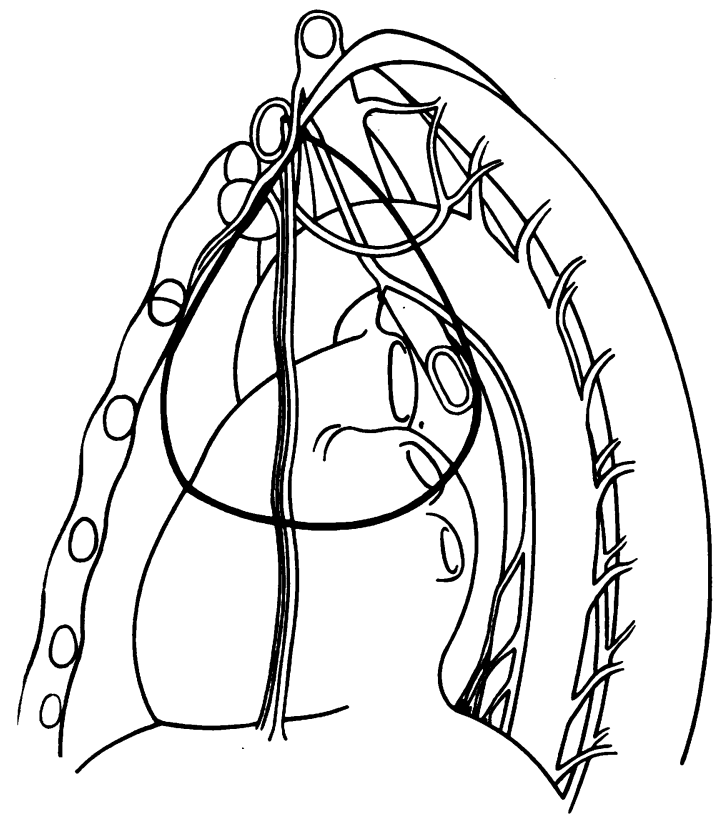

FIG. 2. Diagram of mediastinal structures as viewed from the left side. The thick line indicates the extent of dissection of the parietal pleura.

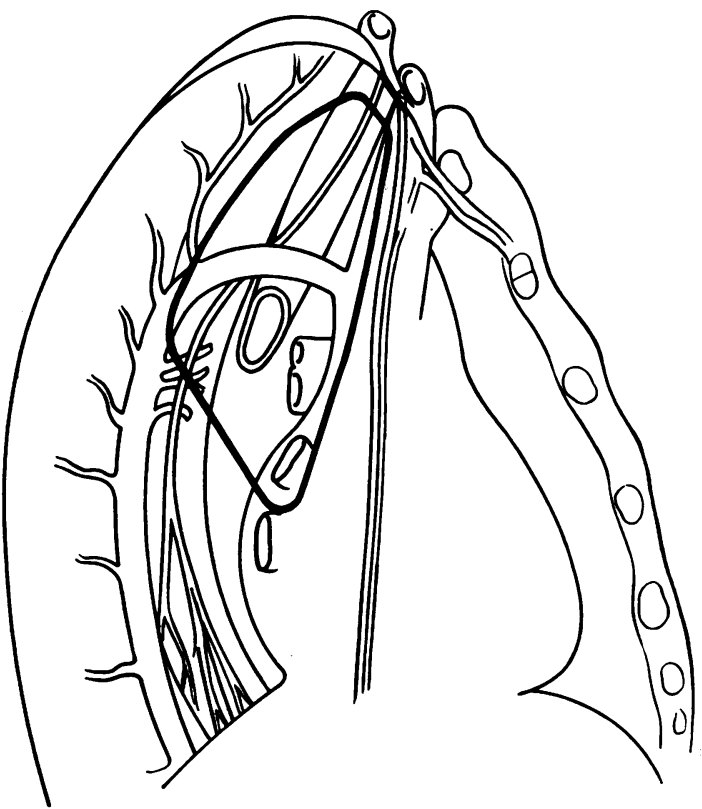

FIG. 3. Diagram of mediastinal structures as viewed from the right side. The thick line indicates the extent of dissection of the parietal pleura. is divided immediately below the main carina and at a lower level, just above the apical segmental division of the lower lobe and above the middle lobe bronchus on the right side. The anastomosis is completed by interrupted atraumatic nylon sutures, the slight discrepancy of the diameter of the upper and lower line of bronchial division being corrected by accurate spacing (Fig. 4). The left-sided anastomosis is constructed posterior to the pulmonary artery, which is held forwards during the anastomosis; this may be done conveniently with a rubber sling. The pulmonary ligament is divided to allow the remainder of the lung to ride up and fill the chest, which is then

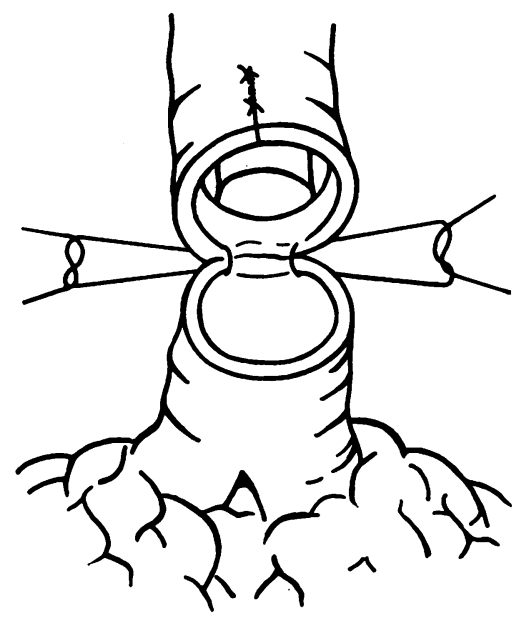

FIG. 4. End-to-end anastomosis is carried out with interrupted nylon sutures.

closed using three fenestrated rubber drains, anterior, posterior, and intermediate. These are connected to wall suction and are removed post-operatively as indicated.

Each resected specimen was examined when the site of origin of the tumour was established (Fig. 5). In certain cases, due to extensive local spread, this was difficult to identify and the site of maximum involvement was recorded. The related lymph nodes were examined for invasion by tumour cells. Twentyseven tumours were related to the right upper lobe orifice and 17 to the left upper lobe orifice. In two cases the carcinoma arose at the origin of the left lower lobe. Here left lower lobectomy with sleeve resection was carried out, with anastomosis of the upper lobe stump to the left main bronchus ('reversed sleeve').

Histologically the main tumour type was squamouscell carcinoma (Table). In addition there were three adenocarcinomas, one chondrosarcoma, and one secondary tumour from a testicular teratoma. 


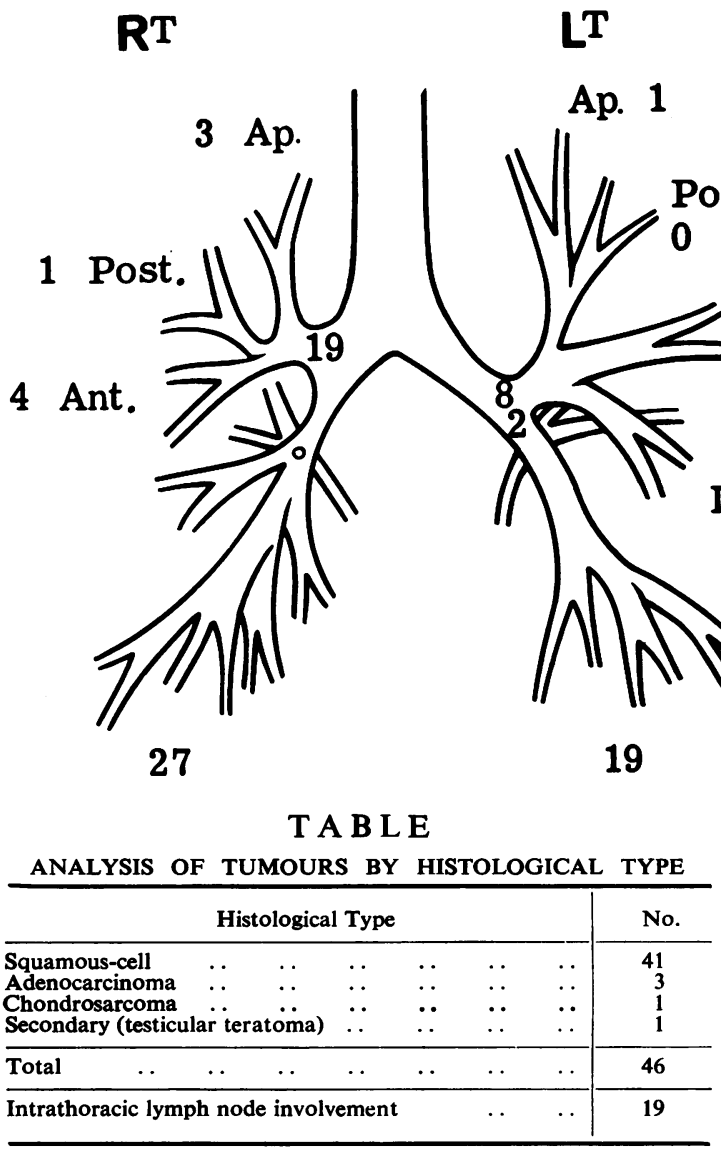

RESULTS

One patient died five days post-operatively from an overwhelming chest infection, giving a hospital mortality of $2 \cdot 2 \%$. In addition, seven other
Ant. 3

Ling. 5
FIG. 5. Diagram to indicate distribution of tumours: 27 originate from the right $\overrightarrow{\overrightarrow{2}}$ side and 19 from the left. $(A p .=$ apical, post. $=$ posterior; ant. $=$ anterior; ling. $=$ or lingular segmental divisions.) patients died within a year of operation, six of carcinomatosis and one of pulmonary embolus five months post-operatively (Fig. 6). Twenty-three patients in all have died since operation, 18 of recurrence, two of bronchopneumonia, one of pulmonary embolus, one of respiratory failure $\frac{3}{3}$ and one of a brain abscess following completion? pneumonectomy. Of a possible 43 patients, 350 $(81 \%)$ lived more than one year after operation (Fig. 7). Of a possible 14 patients, five $(35 \%)$ livedo more than five years following operation. In the 19 patients whose mediastinal nodes containedo malignant cells the survival figures are less satis 3 . factory (Fig. 8). One year after operation 12 outo of $18(66 \%)$ were alive, but no patient is alive five years after surgery.
FIG. 6. Analysis of death rates directly due to operation, at one year and overall mortality.
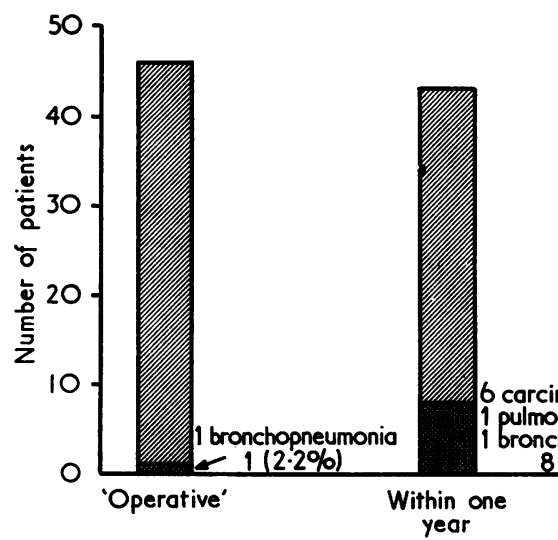

corcinomatosis pulmonary embolus $8(19 \%)$

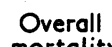
mortality 


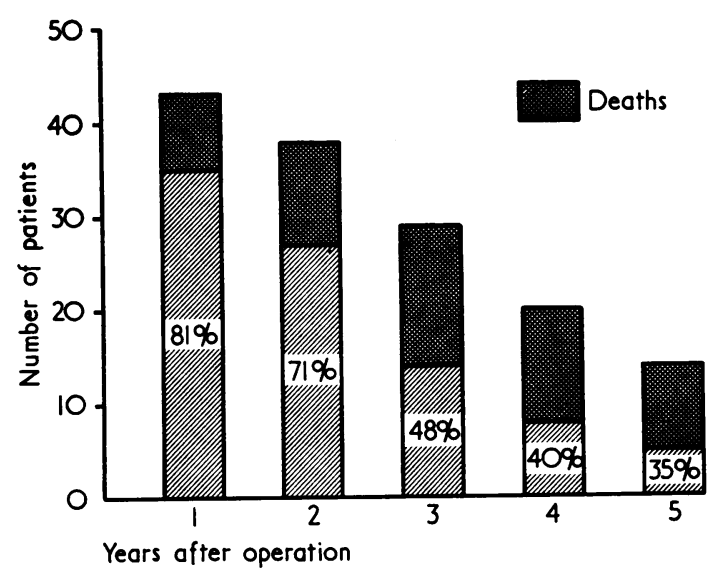

FIG. 7. Survival rates at yearly intervals from one to five years after operation.

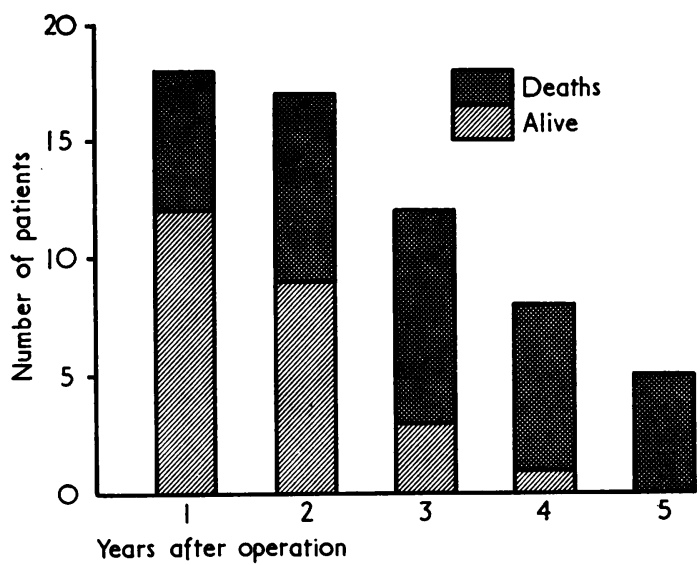

FIG. 8. Survival rates at yearly intervals in the groups of patients with histological proof of tumour invasion of mediastinal glands.
Eight patients (17\%) developed narrowing, proved bronchoscopically, at the bronchial anastomosis due either to kinking caused by expansion of the remainder of the lung to occupy the thoracic cavity or to stenosis. Many of these patients developed recurrent infections distal to the bronchial narrowing and some required repeated dilatation of the anastomosis with aspiration of accumulated secretions. In one case repeated infections caused fibrosis with suppuration, so that completion pneumonectomy became necessary. He died post-operatively from a brain abscess which was confirmed by necropsy, but there was no evidence of carcinoma six years after his sleeve resection. One patient developed local recurrence of carcinoma at the anastomotic site, but bronchial biopsies of other patients with narrowing have remained persistently negative. In addition to eight patients with proved 'strictures', six others have, on clinical examination, a moderately loud fixed rhonchus on the operated side. This may represent bronchial suture line narrowing, but we have no confirmatory bronchoscopic evidence.

Comparison of pre-operative and post-operative $\mathrm{FEV}_{1} / \mathrm{FVC}$ ratios in a random group of six patients confirms that lobectomy with sleeve resection causes little impairment of lung function (Fig. 9). In one case (R. H.), the values were minimally improved, due probably to an associated pre-operative chest infection. There also appears to be little deterioration with the passage of time (in $\mathbf{R} . \mathrm{H}$. the interval between lung function tests was five years, in H.S. over three years, and in G. C. nearly six years).

\section{DISCUSSION}

The first reported use of sleeve resection with lobectomy in the treatment of bronchial carci-

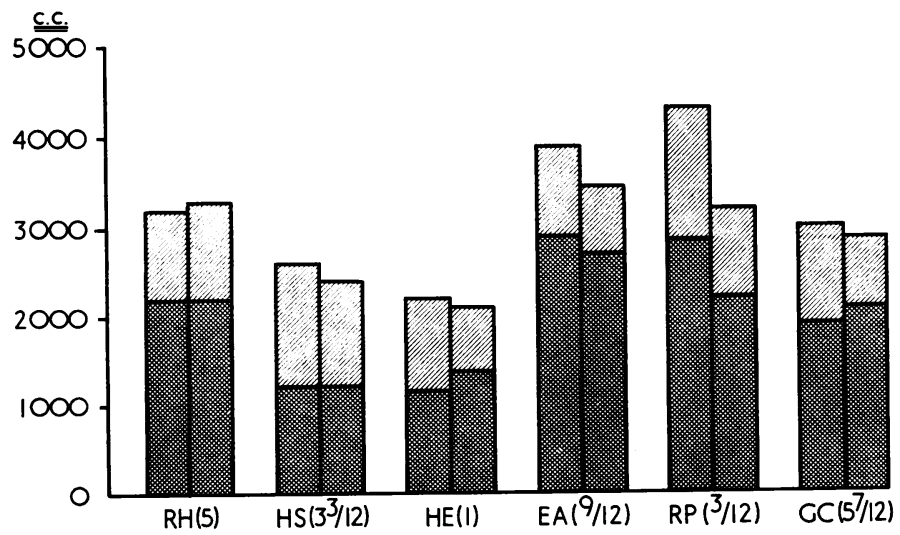

FIG. 9. Pre-operative and post-operative ratio of forced expiratory volume in one second $\left(F E V_{1}\right)$ to forced vital capacity $(F V C)$. The first column indicates preoperative readings and the second postoperative readings. The figures following patients' initials indicate the interval in years and months between recordings. Crosshatched columns $=F E V_{1}$; diagonally hatched columns +crosshatched columns $=F V C$. 
noma was in 1952 (Allison, 1952). Prior to that the procedure has been used to treat non-malignant conditions such as bronchial adenoma and tuberculous stricture (Price Thomas, 1952 ; 1956). Since then others (Johnston and Jones, 1959 ; Le Roux, 1968) have emphasized its merits, but it has by no means achieved general acceptance.

The hazards of pneumonectomy in the older patient are considerable. At centres with wide experience operative mortality figures of $22 \%$ (Price Thomas, quoted by Jones, 1959) were recorded in patients over 60 years of age. The direct comparison of reported mortality rates is clearly of little statistical value due to uncontrolled factors, but it is interesting to compare an overall operative mortality for pneumonectomy carried out by the same surgeon of $6 \%$ and a five-year survival rate of $25 \%$ with an operative mortality rate for sleeve resection of $2.2 \%$ and a five-year survival rate of 35\% (Paneth, 1969).

In selected patients pneumonectomy generally does not cause initial respiratory embarrassment, but in time they may become severely incapacitated by dyspnoea (Price Thomas, 1956). The results of lung function tests obtained from our small randomly selected series of patients confirms that lobectomy with sleeve resection causes little physiological impairment. The advantages of this procedure in patients with poor lung function due to associated pulmonary disease need hardly be stressed.

The quoted survival figures and the fact that in the whole group one patient only developed a recurrence at the site of anastomosis indicate that a satisfactory clearance of tumour is obtained by this technique.
One undoubted disadvantage of sleeve resection is the moderately high incidence of narrowing at the site of bronchial anastomosis causing morbidity in some patients by recurrent infections. This often necessitates bronchoscopy, dilatation, and aspiration. In one instance such infection caused the eventual death of the patient from brain abscess. In the majority, however, the anastomotic technique gives a satisfactory result and it should be noted that no case has developed a bronchopleural fistula, a recognized hazard of pneumonectomy. The operation is technically more difficult and time-consuming than pneumonectomy, but the results would deny that the postoperative period is more difficult to manage or is more hazardous, and the long-term results certainly compare favourably with those of pneumonectomy.

We gratefully acknowledge the invaluable help of the Royal Marsden Hospital Medical Art Department in the preparation of the illustrations.

\section{REFERENCES}

Allison, P. R., at Ass. Thoracic Surgeons meeting, Nov. 1952, quoted by Johnston, J. B., and Jones, P. H. (1959) q.v.

Brock, R., and Whytehead, L. L. (1955). Radical pneumonectomy for bronchial carcinoma. Brit. J. Surg. , 43, 8.

Johnston, J. B., and Jones, P. H. (1959). The treatment of bronchial carcinoma by lobectomy and sleeve resection of the main bronchus. Thorax, 14, 48.

Jones, P. H. (1959). Lobectomy and bronchial anastomosis in the surgery of bronchial carcinoma. Ann. roy. Coll. Surg. Engl., $25,20$.

Le Roux, B. T. (1968). Bronchial Carcinoma, p. 7, p. 36. Livingstone, Edinburgh and London.

Nohl, H. C. (1962). The Spread of Carcinoma of the Bronchus. p. 36. Lloyd-Luke, London.

Paneth, M. (1969). Personal communication.

Price Thomas, C. (1952). Carcinoma of the lung. Ann. roy. Coll. Surg. Engl., 11, 205.

(1956). Conservative resection of the bronchial tree. J. roy. Coll. Surg. Edinb., 1, 169. 